\title{
Caractérisation de bactéries lactiques thermophiles isolées de yaourts artisanaux grecs. I. Souches de Streptococcus salivarius subsp thermophilus
}

\author{
A Zourari 1, S Roger 1, C Chabanet 2, MJ Desmazeaud 1* \\ 1 INRA, station de recherches laitières; \\ 2 station de biométrie, 78352 Jouy-en-Josas Cedex, France
}

(Reçu le 13 février 1991; accepté le 6 mai 1991)

\begin{abstract}
Résumé - Vingt souches de Streptococcus salivarius subsp thermophilus isolées de yaourts artisanaux grecs ont été comparées sur la base des caractères suivants : acidité titrable, viscosité et quantité d'acétaldéhyde produites au cours de la croissance dans le lait; vitesse maximale d'acidification, $\mathrm{pH}$ et temps auxquels la vitesse d'acidification maximale est observée; activité uréasique. Toutes les souches étudiées ont une activité uréasique et présentent deux valeurs maximales de la vitesse d'acidification. Les deux derniers paramètres ne sont pas étroitement corrélés. Cependant, une bonne corrélation est observée entre chacun de ceux-ci, et le temps pendant lequel les valeurs de la vitesse d'acidification sont supérieures à la moitié de la vitesse d'acidification maximale. Les souches étudiées ne produisent pas de viscosité notable dans le lait. La quantité d'acétaldéhyde produite varie entre 2,5 et $6,5 \mathrm{ppm}$. Après conservation à $4{ }^{\circ} \mathrm{C}$ pendant 21 jours, la quantité d'acétaldéhyde est significativement réduite. L'analyse en composantes principales a été utilisée pour grouper les souches. La comparaison de leurs propriétés a été facilitée par la réalisation de graphiques sous forme d'étoiles, représentant simultanément tous les caractères étudiés. Ainsi, une grande diversité des propriétés parmi les souches examinées a été mise en évidence.
\end{abstract}

Streptococcus salivarius subsp thermophilus / yaourt / acidification / acétaldéhyde / analyse en composantes principales

Summary - Characterization of lactic acid bacteria isolated from Greek yogurts. I. Streptococcus salivarius subsp thermophilus strains. Twenty Streptococcus salivarius subsp thermophilus strains isolated from Greek yogurts were compared on the basis of their titratable acidity, viscosity, amount of acetaldehyde produced in milk, maximum acidification rate, corresponding time and $\mathrm{pH}$ value, urease activity. All strains are characterized by $\mathrm{pH}$ kinetics with 2 maximum acidification rates due to their urease activity. These 2 parameters are not closely correlated but a good correlation exists between them and the corresponding time during which the observed acidification rate is higher than half of its maximum value. The strains studied do not produce substantial ropiness in milk. The amount of acetaldehyde produced ranged between 2.5 and $6.5 \mathrm{ppm}$, significantly reduced after storage at $4{ }^{\circ} \mathrm{C}$ for $21 \mathrm{~d}$. Principal component analysis was used for strain grouping. Compari-

\footnotetext{
* Correspondance et tirés à part
} 
son of their properties was facilitated by "star" graphics presenting all properties of each strain simultaneously. A significant diversity between properties of different strains was revealed.

Streptococcus salivarius subsp thermophilus / yogurt / acidification / acetaldehyde / principal component analysis

\section{INTRODUCTION}

Le yaourt est un lait fermenté très populaire dans de nombreux pays d'Europe. II est fabriqué essentiellement à l'échelle industrielle, avec la préoccupation principale d'obtenir régulièrement un produit d'excellente qualité.

Trois facteurs jouent un rôle important dans la qualité du yaourt : le lait utilisé, la technologie de fabrication, et surtout les microorganismes du levain qui appartiennent aux espèces Streptococcus salivarius subsp thermophilus et Lactobacillus delbrueckii subsp bulgaricus. En effet, seul un lait de bonne qualité peut favoriser la croissance des bactéries et conduire à un bon produit (Desmazeaud, 1989). La technologie de fabrication est aussi importante parce qu'elle peut modifier l'activité du levain. Toutefois, sans un levain convenable, rien de cela n'est suffisant : le goût et l'arôme dépendent de l'acide lactique et des composés volatils produits par les bactéries lactiques; les polysaccharides exocellulaires produits par certaines souches assurent une agréable texture et une résistance aux traitements mécaniques des yaourts brassés et liquides (Driessen, 1988).

Autrefois, le yaourt était élaboré à partir d'une microflore naturelle, constituée d'un mélange de souches généralement inconnues, avec des techniques de production très variables. Aujourd'hui, des chaînes de production complexes sont utilisées pour obtenir des produits qui satisfont le consommateur. Cependant, cette évolution a conduĭt à un appauvrissement de la microflore des yaourts industriels. En effet, un nombre réduit de souches sont utilisées, cultivées depuis longtemps hors de la compétition de l'environnement naturel (Kurmann, 1984). Dans le but d'enrichir les levains industriels avec des souches ayant des propriétés intéressantes du point de vue technologique et diététique, la recherche s'oriente actuellement vers la mise en place de collections de bactéries lactiques issues de produits artisanaux ou traditionnels de bonne qualité.

Depuis les travaux de Bouillanne et Desmazeaud (1980, 1981), de nouvelles méthodes d'évaluation de l'activité acidifiante des souches sont apparues et l'influence de l'activité uréasique dans le suivi de l'acidification dans le lait a été mise en relief (Juillard et al, 1988; Spinnler et Corrieu, 1989). Des techniques performantes facilitent l'évaluation de la production de composés d'arôme (Degorce-Dumas et al, 1986) et l'étude des polysaccharides exocellulaires produits par les bactéries lactiques a donné ses premiers résultats (Cerning et al, 1986, 1988). Nous avons ainsi caractérisé différentes souches de $S$ salivarius subsp thermophilus et de $L$ delbrueckii subsp bulgaricus utilisées dans la fabrication de yaourts artisanaux grecs de bonne qualité organoleptique, en utilisant des critères d'intérêt technologique : acidification du lait (suivi de l'acidité titrable et du $\mathrm{pH}$ au cours de la croissance), viscosité développée dans le lait, production de composés d'arôme. Ce premier article concerne la caractérisation des souches de $S$ salivarius subsp thermophilus. II sera suivi d'un deuxième article décrivant les propriétés de différentes souches de $L$ del- 
brueckii subsp bulgaricus et l'étude du comportement de quelques souches appartenant à ces deux espèces, en coculture.

\section{MATÉRIEL ET MÉTHODES}

\section{Souches et conditions de culture}

Vingt souches de $S$ salivarius subsp thermophilus isolées à partir de yaourts artisanaux grecs (Zourari, 1991) ont été étudiées (tableau I). Nous y avons joint la souche-type de $S$ salivarius subsp thermophilus, ATCC 19258, et les souches CNRZ 302, 385, 404 et 407, qui ont des propriétés particulières.
Les souches sont conservées à $-20^{\circ} \mathrm{C}$ dans du lait tournesolé (lait additionné de teinture de tournesol (Litmus, Difco) à $0,075 \%$, stérilisé à $118^{\circ} \mathrm{C}$ pendant $\left.15 \mathrm{~min}\right)$. Pour la préparation des précultures, le lait est inoculé à $10 \%$ avec la suspension des cellules dans le lait tournesolé et incubé par la suite à $42{ }^{\circ} \mathrm{C}$ pendant environ $12 \mathrm{~h}$. Cette préculture sert à l'inoculation des cultures de travail (à $3 \%$ ), dans du lait préparé avec de la poudre de lait écrémé exempte d'antibiotiques (Elle \& Vire, France), reconstitué à $10 \%$ d'extrait sec et stérilisé à $110^{\circ} \mathrm{C}$ pendant $10 \mathrm{~min}$. L'incubation se fait toujours à $42^{\circ} \mathrm{C}$.

\section{Acidité titrable}

Les mesures de l'acidité titrable au cours de la croissance dans le lait sont effectuées selon la méthode décrite par Accolas et al (1977). L'aci-

Tableau I. Souches de $S$ salivarius subsp thermophilus étudiées et leur origine.

$\mathrm{S}$ salivarius subsp thermophilus strains and their origin.

Souches * Origine

CNRZ 1156 (1), CNRZ 1197 (5),

CNRZ 1201 (6), CNRZ 1206 (3),

CNRZ 1207 (4), CNRZ 1208 (4),

CNRZ 1237 (2)

CNRZ 1157 (11), CNRZ 1198 (7),

CNRZ 1199 (8), CNRZ 1200 (11),

CNRZ 1202 (8), CNRZ 1203 (9),

CNRZ 1204 (10), CNRZ 1205 (11),

CNRZ 1209 (7), CNRZ 1210 (7),

CNRZ 1211 (8), CNRZ 1212 (8),

CNRZ $1213(8)$

ATCC 19258 (NCDO 573)

CNRZ 302

CNRZ 385

CNRZ 404

CNRZ 407
Yaourts artisanaux grecs, région de Hania (Crète)

Yaourts artisanaux grecs, région d'Héraclion (Crète)

Souche-type

Levain artisanal de Gruyère de Comté (Jura, France)

Yaourt sucré (Japon)

Levain commercial mixte (France)

Levain commercial pour yaourt (France)

* Entre parenthèses, le numéro de l'échantillon de yaourt à partir duquel la souche a été isolée (Zourari, 1991).

* In parentheses, number of yogurt sample from which strain has been isolated (Zourari, 1991). 
dité titrable juste après l'inoculation est soustraite aux valeurs de l'acidité mesurées au cours de la croissance pour obtenir l'acidité réellement produite (acidité acquise).

\section{Suivi du pH au cours de la croissance}

La méthode décrite par Corrieu et al (1988) et par Spinnler et Corrieu (1989) est utilisée. La suspension cellulaire, conservée à $-20^{\circ} \mathrm{C}$, est décongelée et incubée à $42^{\circ} \mathrm{C}$ jusqu'à la coagulation du lait, et sert à l'inoculation de $150 \mathrm{ml}$ de lait (à 1\%). Celui-ci est alors incubé à $42^{\circ} \mathrm{C}$ sous agitation continue, pour la mesure du $\mathrm{pH}$.

La moyenne des valeurs obtenues en $120 \mathrm{~s}$ est enregistrée. Les vitesses d'acidification (dérivées $\mathrm{dpH} / \mathrm{dt}$ ) sont calculées (pente entre le point précédent et le point saisi) et exprimées en milliunités de $\mathrm{pH}$ par min $\left(\mathrm{mU} \mathrm{pH} \cdot \mathrm{min}^{-1}\right)$. Les paramètres estimés pour caractériser les souches sont :

- la vitesse maximale d'acidification (Vm) exprimée en $\mathrm{mU} \mathrm{pH} \cdot \mathrm{min}^{-1}$;

- le pH ( $p H m)$ et le temps (Tm, en min) auxquels la $V m$ est observée;

- la plage de $\mathrm{pH}(p H 50)$ et le temps (T5O, en $\mathrm{min}$ ) pendant lesquels la vitesse d'acidification est supérieure à $\mathrm{V} / 2$.

\section{Viscosité développée dans le lait}

La viscosité développée dans le lait est mesurée après incubation jusqu'à $\mathrm{pH} 5,4,7$ et 4,5 $( \pm 0,05)$, au moyen d'un viscosimètre rotatif à cylindres coaxiaux (appareil Rotovisco RV 12, Haake, Allemagne), muni d'un dispositif de mesure du type NV (à double fente). Les conditions de mesure sont les suivantes : gradient de vitesse $173 \mathrm{~s}^{-1}$, température $42{ }^{\circ} \mathrm{C}, 10 \mathrm{ml}$ d'échantillon. Par ailleurs, la viscosité et le pH sont mesurés après conservation des cultures à $4^{\circ} \mathrm{C}$ pendant 21 jours ( $\mathrm{pH}$ initial de 4,5 ).

\section{Dosage de l'acétaldéhyde produit dans le lait}

Les souches sont incubées dans le lait jusqu'à $\mathrm{pH} 5( \pm 0,05)$. A ce pH, un échantillon est préle- vé pour le dosage de l'acétaldéhyde, tandis qu'un autre est conservé à $4^{\circ} \mathrm{C}$ pendant 21 jours pour un nouveau dosage de l'acétaldéhyde et une mesure du $\mathrm{pH}$.

Une méthode basée sur l'analyse de l'espace de tête (head-space) par chromatographie en phase gazeuse, décrite par Degorce-Dumas et al (1986) est utilisée, mais au lieu d'un couplage direct head-space / colonne chromatographique, un prélèvement manuel de la phase vapeur est effectué. Dans $2 \mathrm{ml}$ d'échantillon, $1 \mathrm{ml}$ d'acide sulfurique $1 \mathrm{M}$ saturé en sulfate d'ammonium $\left[\left(\mathrm{NH}_{4}\right)_{2} \mathrm{SO}_{4}\right]$ et $0,7 \pm 0,1 \mathrm{~g}$ de $\left(\mathrm{NH}_{4}\right)_{2} \mathrm{SO}_{4}$ sont ajoutés, dans des flacons étanches. Après chauffage à $70^{\circ} \mathrm{C}$ pendant $20 \mathrm{~min}$ sous forte agitation continue (agitateur magnétique), $300 \mu$ l de phase vapeur sont prélevés au moyen d'une seringue étanche à gaz, et injectés pour l'analyse par chromatographie en phase gazeuse.

Un chromatographe Girdel 3000 (Delsi, Suresnes, France) est utilisé. Il est équipé d'un détecteur à ionisation de flamme et d'une colonne inox ( $3 \mathrm{~m}, 1 / 8$ de pouce) remplie de Chromosorb GAW-DMCS 80/100 mesh imprégné à $10 \%$ de Carbowax $20 \mathrm{M}$, TPA (phase polaire). Les conditions chromatographiques sont les suivantes : gaz vecteur azote, débit $17 \mathrm{ml} \cdot \mathrm{min}^{-1}$; température de l'injecteur $180^{\circ} \mathrm{C}$; température du détecteur $180^{\circ} \mathrm{C}$; température de colonne $40^{\circ} \mathrm{C}$ (isotherme). L'acétaldéhyde est identifié à partir de son temps de rétention.

\section{Dosage de l'activité uréasique}

Le dosage est effectué selon la méthode décrite par Juillard et al (1988), avec des cellules récoltées au début de la phase stationnaire, après croissance dans le milieu M17 (Terzaghi et Sandine, 1975) contenant du lactose $(10 \%)$ et de l'urée $(25 \mathrm{mM})$. l'activité uréasique est exprimée en $\mu \mathrm{mol}$ d'urée dégradée par min et par $\mathrm{mg}$ de poids sec des cellules $\left(\mu \mathrm{mol} \cdot \mathrm{min}^{-1} \bullet \mathrm{mg}^{-1}\right.$ ).

\section{Analyse des données}

Une analyse exploratoire des données est appliquée. Elle est basée sur différentes représentations graphiques et complétée par une analyse 
en composantes principales. Pour cela, le logiciel S (New S Language), qui fonctionne sur le système Unix, est utilisé (Becker et al, 1988).

\section{RÉSULTATS}

\section{Acidification du lait}

\section{Acidité titrable acquise}

Les 20 souches étudiées acidifient moins que la souche CNRZ 385, connue pour ses propriétés d'acidification (Accolas et al, 1977; Bouillanne et Desmazeaud, 1980). II y a beaucoup de similitudes entre les profils d'acidification des différentes souches, sur la base de l'acidité titrable après 2, 4, 6 ou $24 \mathrm{~h}$ d'incubation (fig 1). Les différences concernent notamment la présence ou non d'une phase de latence au début de l'acidification. Après 24 h d'incubation, l'acidité titrable atteint des valeurs qui varient, selon la souche, entre 0,5 (souche CNRZ 302) et $0,65 \%$ d'acide lactique (souches CNRZ $1199,1205,1206)$, à l'exception des souches CNRZ 1157 et 1200, qui produisent plus de $0,7 \%$ d'acide lactique.

\section{Suivi du $\mathrm{pH}$}

La vitesse d'acidification des streptocoques se caractérise par deux valeurs maximales, $V m_{1}$ et $V m_{2}$ (fig 2). La valeur de $V m_{1}$ est observée à $T m_{1}$ entre 35 et 87 min pour la majorité des souches (entre 103 et 130 pour quatre souches). Les valeur's de $\mathrm{pHm}_{1}$ sont comprises entre 5,8 et 6,3 . Ensuite, une stabilisation $\mathrm{du} \mathrm{pH}$ est observée, voire même une légère augmentation (caractérisant par exemple la souche ATCC 19258). La valeur de $V m_{2}$ apparaît à $T m_{2}$ entre 80 et $265 \min$ (364 min pour la souche-type ATCC 19258).
Les valeurs de $\mathrm{pH} m_{2}$ varient entre 5,5 et 6. En général, une grande valeur de $V m_{1}$ est suivie d'une grande valeur de $V / m_{2}$, sans qu'une corrélation linéaire étroite puisse être établie entre les deux paramètres. Les souches CNRZ 1156 et 1199 se caractérisent par les valeurs de $V m_{1}$ et $V m_{2}$ les plus élevées parmi les souches étudiées (entre 12,4 et $13,8 \mathrm{mU} \mathrm{pH} \cdot \mathrm{min}^{-1}$ ). La souche ATCC 19258 a les vitesses d'acidification les plus faibles $(3,3$ et $2,6 \mathrm{mU}$ $\mathrm{pH} \cdot \mathrm{min}^{-1}$, pour $V m_{1}$ et $V m_{2}$, respectivement).

\section{Post-acidification}

Conservées à $\mathrm{pH}$ initial égal à 5 , les souches provoquent en général une acidification supplémentaire du lait après 21 jours à $4{ }^{\circ} \mathrm{C}$, qui est inférieure à 0,35 unité de $\mathrm{pH}$. Si le $\mathrm{pH}$ au début de la conservation est de 4,5 , les souches ne modifient pas cette valeur.

\section{Viscosité développée dans le lait}

Le caractère épaississant des souches de $S$ salivarius subsp thermophilus étudiées demeure faible et n'est guère intéressant pour la fabrication de yaourt. $\grave{A} \mathrm{pH} 5$, la viscosité varie entre 40 et $60 \mathrm{mPa} \cdot \mathrm{s}$. À pH 4,5 , elle atteint des valeurs comprises entre 75 et $105 \mathrm{mPa} \cdot \mathrm{s}$. Après 21 jours à $4^{\circ} \mathrm{C}$, la viscosité augmente légèrement.

\section{Production d'acétaldéhyde dans le lait}

Dans le lait acidifié à $\mathrm{pH} 5$, les souches étudiées produisent entre 2,5 et $6,5 \mathrm{ppm}$ d'acétaldéhyde. Ces quantités sont inférieures à celle qui est produite par la souche CNRZ 385 (10,5 ppm), mais bien 
supérieures à celle de la souche-type ATCC 19258, qui produit moins de 2 ppm.

Après 21 jours à $4^{\circ} \mathrm{C}$, la quantité d'acétaldéhyde diminue d'environ un tiers. Elle est, en général, comprise entre 2 et 5 ppm (1,5 ppm pour la souche-type et $6 \mathrm{ppm}$ pour la souche CNRZ 385).

\section{Activité uréasique}

Les 20 souches de $S$ salivarius subsp thermophilus isolées des yaourts grecs possèdent une activité uréasique qui varie entre 0,3 et $2 \mu \mathrm{mol} \cdot \mathrm{min}^{-1} \cdot \mathrm{mg}^{-1}$. Les souches CNRZ 1209, 1210 et la souche type, ATCC 19258, ont les activités uré-asiques les plus fortes, d'envion $2 \mu \mathrm{mol} \cdot \mathrm{min}^{-1} \cdot \mathrm{mg}^{-1}$. Les autres souches ont des activités inférieures à $1,4 \mu \mathrm{mol} \cdot \mathrm{min}^{-1} \cdot \mathrm{mg}^{-1}$. Parmi les 4 souches de la collection CNRZ utilisées comme référence, les souches 302,385 et 404 ont une activité uréasique qui varie entre 1,1 et $1,6 \mu \mathrm{mol} \cdot \mathrm{min}^{-1} \cdot \mathrm{mg}^{-1}$, alors que la souche CNRZ 407 en est dépourvue.

\section{Analyse des données}

Dans un premier temps, les relations existant entre les variables utilisées sont recherchées. Par la suite, une analyse en composantes principales est appliquée afin de classer les souches en tenant compte simultanément de tous les caractères étudiés.

\section{Relations entre les variables}

\section{Variables relatives à l'acidification du lait}

La recherche des relations entre les différentes variables estimées par le suivi automatique du $\mathrm{pH}\left(\mathrm{Vm}_{1,2}, \mathrm{pH} m_{1,2}, T m_{1,2}\right.$, $\left.T 50_{1,2}, \mathrm{pH}_{50} \mathrm{1}_{1,2}\right)$ est particulièrement intéressante, car la présence de deux $\mathrm{Vm}$ peut amplifier la discrimination entre les souches. Les coefficients de corrélation entre les différentes variables sont calculés en utilisant non seulement les valeurs moyennes caractérisant les souches $\left(r_{m}\right.$; tableau II), mais aussi la totalité des observations obtenues pendant les 2 répétitions du suivi du $\mathrm{pH}\left(r_{t} ;\right.$ valeurs non montrées). Des représentations graphiques de ces variables par paires (non montrées) sont réalisées en parallèle pour compléter les comparaisons.

II n'y a pas de corrélation étroite entre $V m_{1}$ et $V m_{2} ;$ le $r_{m}$ étant égal à 0,5 et le $r_{t}$ à 0,4 . Une explication possible serait l'intervention de l'activité uréasique puisque la présence de deux $\mathrm{Vm}$ est due à l'alcalinisation du milieu par l'ammoniaque, résultant de la dégradation de l'urée contenue dans le lait (Juillard et al, 1988; Spinnler et Corrieu, 1989). Cette activité enzymatique varie d'une souche à l'autre, ainsi qu'au cours de la croissance, elle influence donc différemment les deux vitesses d'acidification maximales. Cependant, le niveau de cette activité enzymatique ne peut pas être corrélé aux paramètres de l'acidification, en particulier à $\mathrm{Vm}$ et $\mathrm{pHm}$ (tableau II). La

Fig 1. Acidité titrable acquise développée après $2,4,6$ et $24 \mathrm{~h}$ d'incubation dans le laít, à $42{ }^{\circ} \mathrm{C}$, par les souches de $S$ salivarius subsp thermophilus. Quand une valeur n'est pas déterminée, la souche correspondante n'apparaît pas sur la figure.

Acquired titratable acidity after $2,4,6$ and $24 \mathrm{~h}$ incubation in milk, at $42^{\circ} \mathrm{C}$ for $\mathrm{S}$ salivarius subsp thermophilus strains. If acidity has not been determined, the corresponding strains are not presented in the graphics. 


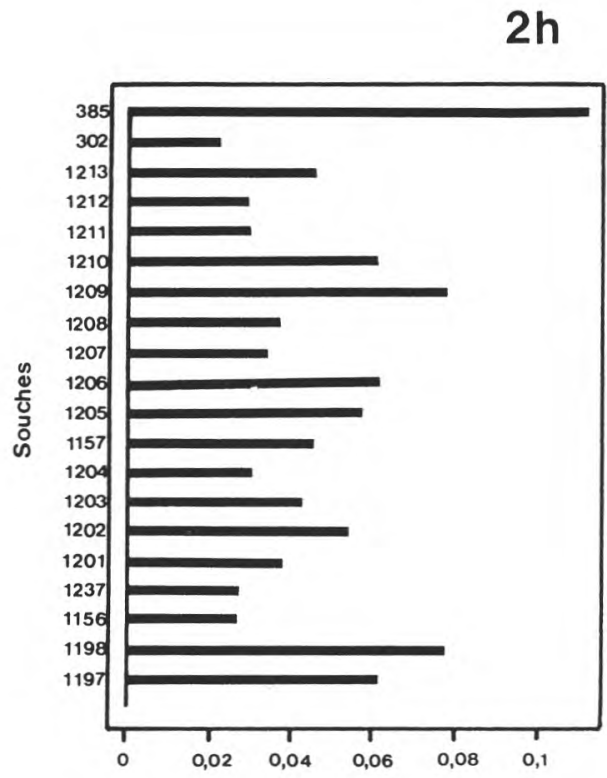

Acidité titrable (\%acide lactique)

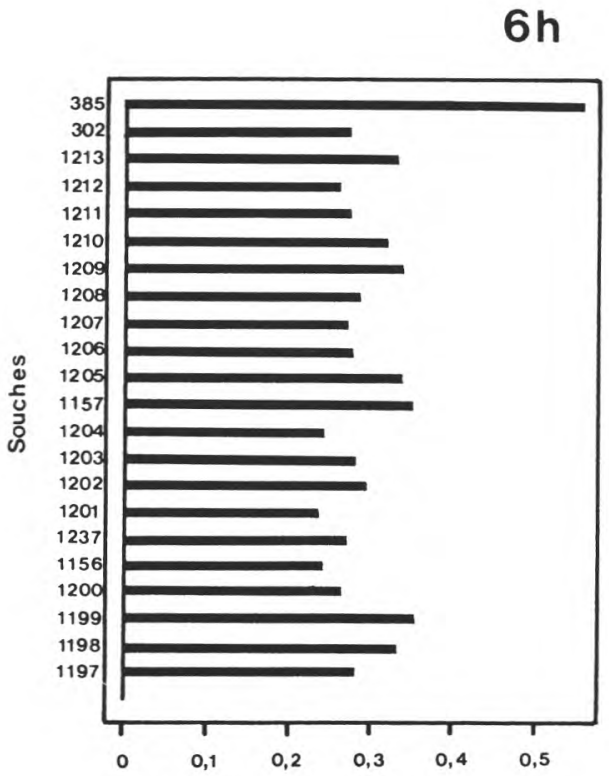

Acidité titrable (\%acide lactique)

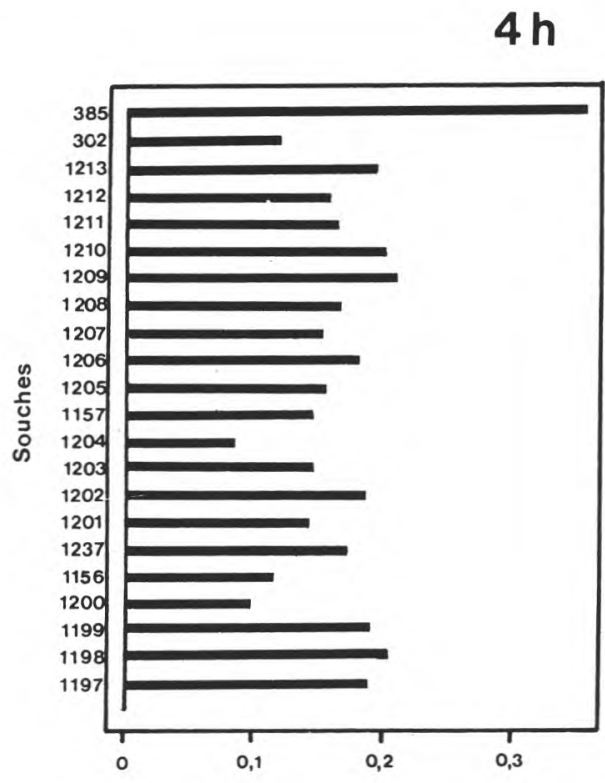

Acidité titrable (\%acide lactique)

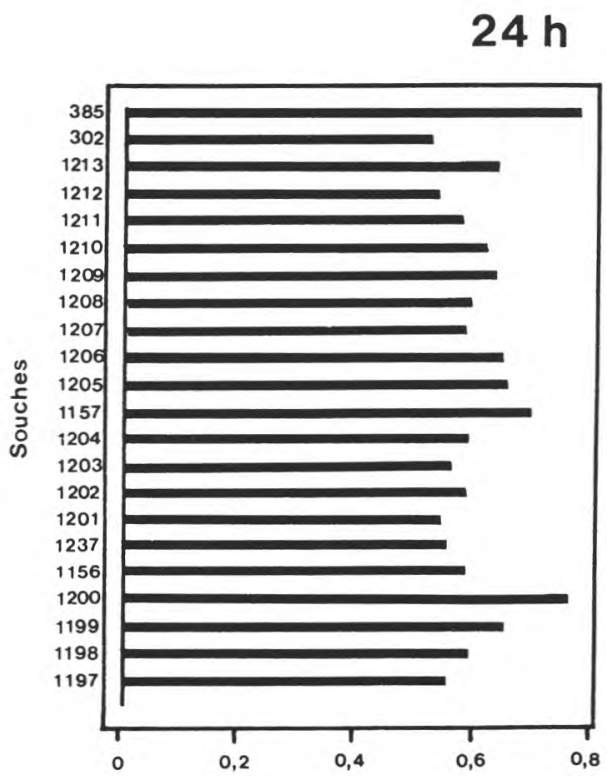

Acidité titrable (\%acide lactique) 


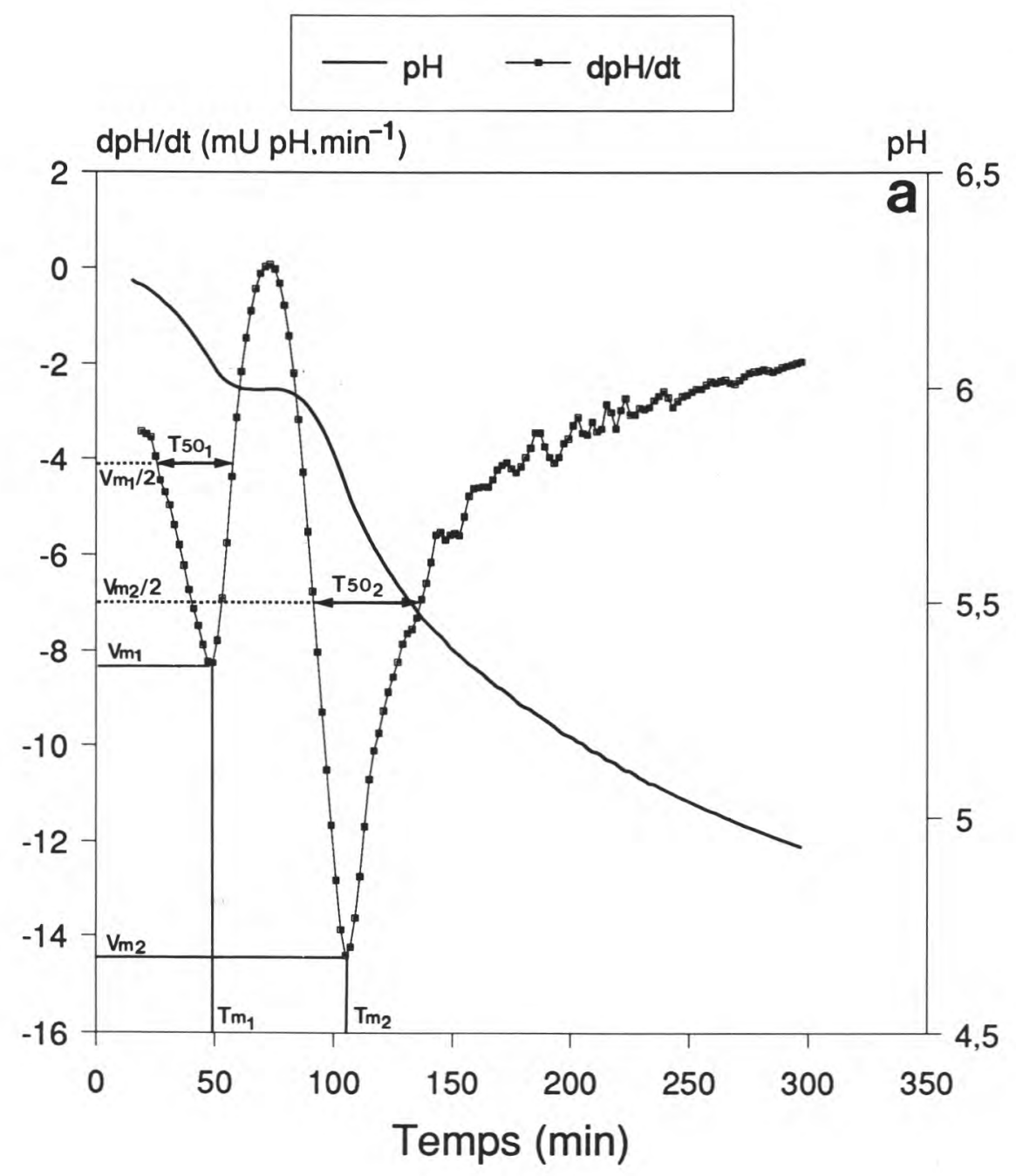

très faible corrélation observée entre l'activité uréasique et $T m_{1}$ ou $T m_{2}$ (tableau II) ne permet pas de conclusions définitives.

Une variable supplémentaire est introduite : le temps $d T m$, égal à $T m_{2}-T m_{1}$. Une forte alcalinisation du milieu devrait à la fois réduire les valeurs de $\mathrm{Vm}$ observées et augmenter celles de $d T m$ et $T 50$. Effectivement, il y a une corrélation négative (tableau II) entre $d T m$ et $V m_{1}$ ou $V m_{2}$ (faible) et une corrélation positive entre $d T m$ et $T 50_{2}$, mais il existe également une très faible corrélation négative entre $d T m$ et l'activité uréasique.

La variable $V m_{1}$ est négativement corrélée avec $T m_{1}, p H m_{1}, T 50_{1}$ et positivement corrélée avec $p H 50_{1}$. De même, une corrélation négative existe entre $V m_{2}$ et $\mathrm{Tm}_{2}$ ou $T 5 \mathrm{O}_{2}$, très forte pour cette dernière variable (tableau II). Des relations existent également entre les temps $\left(T m_{1}, T m_{2}, T 50_{1}\right.$, T502) et les $\mathrm{pH}\left(\mathrm{pHm}, \mathrm{pHm} m_{2}, \mathrm{pH} 50_{1}\right.$, 


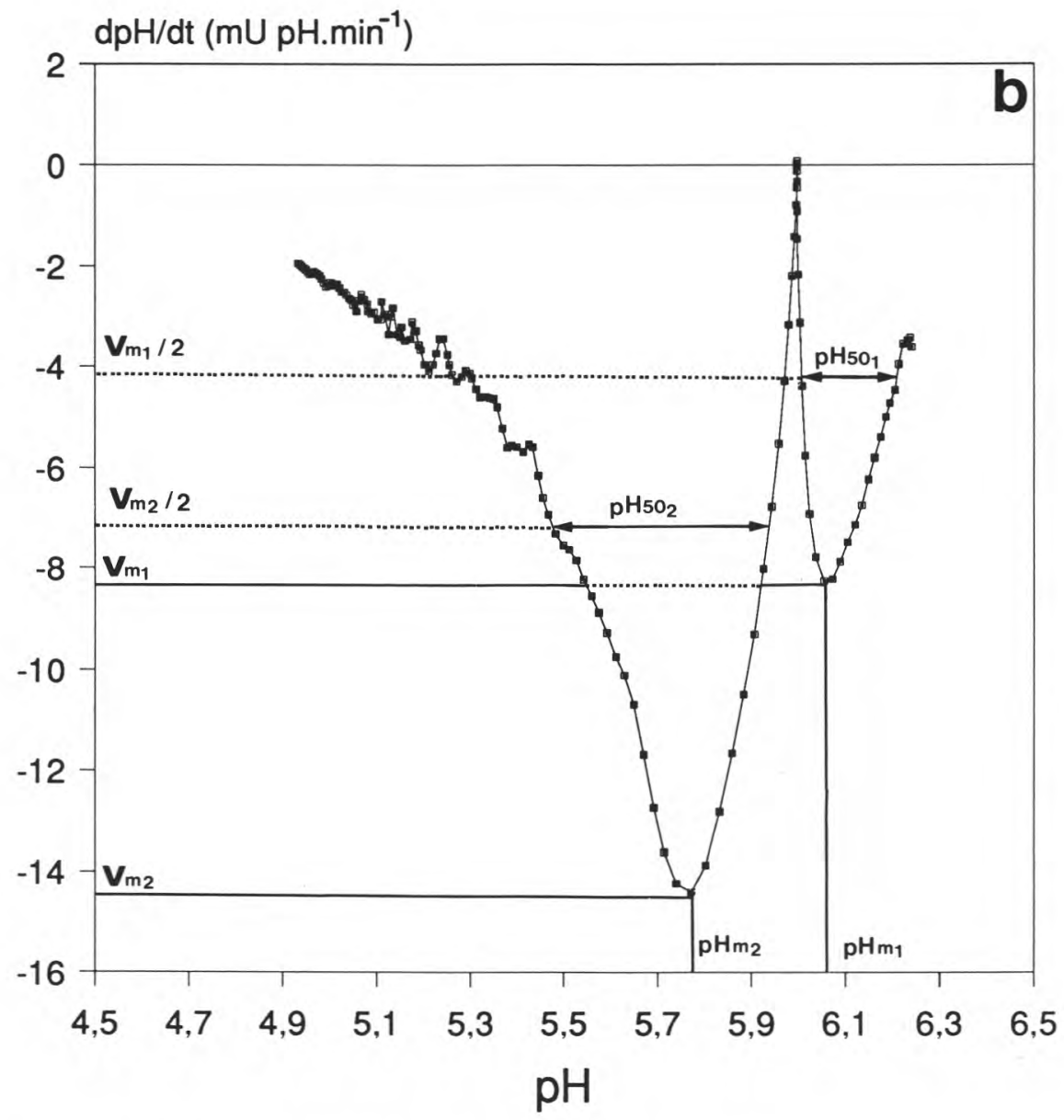

Fig 2. Évolution du pH et de la vitesse d'acidification (dpH/dt), en fonction du temps (a) ou du pH (b) pour la souche CNRZ 1209, caractéristique des souches de $S$ salivarius subsp thermophilus étudiées.

$p H$ and acidification rate (dpH/dt) kinetics versus time (a) or $p H$ (b) for strain CNRZ 1209, characteristic for S salivarius subsp thermophilus strains.

$\mathrm{pH} \mathrm{O}_{2}$ ) caractéristiques des souches, notamment entre $T m_{1}$ et $T m_{2}$ ainsi qu'entre $p H m_{1}$ et $p H m_{2}$, qui sont positivement corrélés (tableau II).

Par ailleurs, aucune corrélation n'est mise en évidence entre les paramètres du suivi automatique du $\mathrm{pH}$ et la chute du $\mathrm{pH}$ après conservation $(d p H)$ ou l'acidité titrable à différents temps d'incubation, en particulier après 2, 4, 6 et $24 \mathrm{~h}$ (tableau II).

II n'est pas possible de mettre en évidence des groupes de souches qui ont un 
Tableau II. Coefficients de corrélation estimés pour les variables relatives à l'acidification du lait pour les souches de $S$ salivarius subsp thermophilus. AT: acidité titrable; $\mathrm{dpH}$ : réduction du pH après 21 jours à $4^{\circ} \mathrm{C} ; d T m=T m_{2}-T m_{1}$.

Correlation coefficients estimated for variables related on milk acidification by $S$ salivarius subsp thermophilus strains. AT: titratable acidity; $d p H$ : $p H$ reduction after 21 days at $4{ }^{\circ} \mathrm{C} ; \mathrm{dTm}=\mathrm{Tm}_{2}-\mathrm{Tm}_{1}$.

\begin{tabular}{|c|c|c|c|c|c|c|c|c|c|c|c|c|c|c|c|}
\hline$V m_{2}$ & 0,47 & & & & & & & & & & & & & & \\
\hline$p H m_{1}$ & $-0,66$ & $-0,51$ & & & & & & & & & & & & & \\
\hline$p H m_{2}$ & $-0,62$ & $-0,54$ & 0,92 & & & & & & & & & & & & \\
\hline $\mathrm{T}^{5 \mathrm{O}_{1}}$ & $-0,67$ & $-0,63$ & 0,56 & 0,58 & & & & & & & & & & & \\
\hline $\mathrm{T} \mathrm{O}_{2}$ & $-0,42$ & $-0,84$ & 0,58 & 0,54 & 0,55 & & & & & & & & & & \\
\hline $\mathrm{pH} \mathrm{O}_{1}$ & 0,65 & 0,03 & $-0,14$ & $-0,12$ & $-0,08$ & 0,04 & & & & & & & & & \\
\hline $\mathrm{pH} \mathrm{O}_{2}$ & $-0,21$ & $-0,12$ & $-0,02$ & $-0,16$ & $-0,14$ & 0,41 & $-0,25$ & & & & & & & & \\
\hline$T m_{1}$ & $-0,71$ & $-0,35$ & 0,37 & 0,27 & 0,66 & 0,43 & $-0,34$ & 0,17 & & & & & & & \\
\hline$T m_{2}$ & $-0,79$ & $-0,49$ & 0,51 & 0,40 & 0,76 & 0,62 & $-0,36$ & 0,35 & 0,90 & & & & & & \\
\hline AT4h & $-0,04$ & $-0,27$ & 0,44 & 0,29 & 0 & 0,21 & 0,13 & $-0,16$ & 0,09 & 0,01 & & & & & \\
\hline AT6h & 0,14 & 0,01 & 0,28 & 0,19 & 0,01 & 0,04 & 0,31 & $-0,33$ & 0,15 & $-0,02$ & 0,61 & & & & \\
\hline AT24h & $-0,22$ & $-0,01$ & 0,27 & 0,16 & 0,21 & 0,20 & $-0,14$ & 0,12 & 0,36 & 0,36 & $-0,16$ & 0,46 & & & \\
\hline$d p H$ & $-0,42$ & $-0,18$ & 0,23 & 0,23 & 0,15 & 0,15 & $-0,37$ & 0,34 & 0,13 & 0,24 & $-0,04$ & $-0,12$ & $-0,01$ & & \\
\hline uréase & 0,21 & 0,23 & $-0,04$ & $-0,13$ & $-0,55$ & $-0,34$ & $-0,12$ & $-0,18$ & $-0,45$ & $-0,53$ & 0,35 & 0,10 & $-0,19$ & 0,09 & \\
\hline$d T m$ & $-0,74$ & $-0,53$ & 0,55 & 0,44 & 0,74 & 0,68 & $-0,32$ & 0,45 & 0,69 & 0,93 & $-0,07$ & $-0,16$ & 0,31 & 0,30 & $-0,52$ \\
\hline & $V m_{1}$ & $V m_{2}$ & $p H m_{1}$ & $p \mathrm{Hm}_{2}$ & T50, & $\mathrm{T} 5 \mathrm{O}_{2}$ & $\mathrm{pH} 5 \mathrm{O}_{1}$ & $\mathrm{pH} 5 \mathrm{O}_{2}$ & $T m_{1}$ & $T m_{2}$ & AT4h & AT6h & AT24h & $d p H$ & uréase \\
\hline
\end{tabular}


comportement similaire. La souche-type ATCC 19258 se trouve toujours à l'écart des autres streptocoques étudiés. Elle se caractérise par des valeurs de $\mathrm{Vm}$ très faibles, de $T m$ et $T 50$ très élevées, et par une activité uréasique importante.

\section{Variables relatives à la viscosité développée dans le lait}

La viscosité mesurée à $\mathrm{pH} 5,4,7$ et 4,5 a été comparée avec la viscosité et le $\mathrm{pH}$ mesurés après 21 jours à $4{ }^{\circ} \mathrm{C}(\mathrm{pH}$ initial de 4,5 ). Seules les viscosités mesurées à $\mathrm{pH} 4,7$, à $\mathrm{pH} 4,5$ et après conservation sont faiblement corrélées entre elles ( $r=$ $0,5)$.

\section{Variables relatives à la production d'acétaldéhyde dans le lait}

Une corrélation positive est observée entre la quantité d'acétaldéhyde produite après culture à $42^{\circ} \mathrm{C}$ (à pH 5) et la quantité mesurée après conservation pendant 21 jours à $4{ }^{\circ} \mathrm{C}(r=0,78)$. II n'y a pas de corrélation entre ces 2 variables, et le $\mathrm{pH}$ après conservation à $4{ }^{\circ} \mathrm{C}$ ( $r$ égal respectivement à 0,43 et 0,09 ).

\section{Analyse en composantes principales (ACP)}

Pour l'ACP, les variables $V m_{1}, V m_{2}, p H m_{1}$, $T m_{1}, T 5 \mathrm{O}_{2}$, acidité titrable après $4 \mathrm{~h}$ de culture, activité uréasique, viscosité à $\mathrm{pH} 4,5$ et acétaldéhyde produit à $\mathrm{pH} 5$ sont prises en compte.

Les deux premiers axes $\left(F_{1}, F_{2}\right)$ ensemble expliquent $58 \%$ de la variance. $\mathrm{Ce}$ taux monte à $72,4 \%$ si l'on y associe l'axe $F_{3}$. La projection du nuage des points représentant les souches étudiées et la position des variables utilisées sur les plans $F_{1} F_{2}$ et $F_{2} F_{3}$ est présenté dans la figure 3 .
L'axe $F_{1}$ met en opposition d'un côté les variables $V m_{1}, V m_{2}$ et de l'autre côté les variables $T m_{1}, T 50_{2}, p H m_{1}$. L'axe $F_{2}$ permet de différencier les souches, surtout sur la base de leur activité uréasique, et de leur acidité titrable après $4 \mathrm{~h}$ de culture. En revanche, le long de l'axe $F_{3}$, c'est surtout la production d'acétaldéhyde qui discrimine les souches.

Des groupes de souches ne sont pas aisément distingués. La souche CNRZ 1198 se trouve à l'écart des autres, à cause de ses valeurs élevées de $\mathrm{pH} m_{1}$ et d'acidité titrable. Sa production d'acétaldéhyde est aussi très élevée. II en va de même pour la souche CNRZ 1200, en raison des valeurs de $T m_{1}$ élevées et des faibles valeurs de $V m_{1}$, de $V m_{2}$, de l'activité uréasique et de l'acidité titrable. Les souches CNRZ 1209 et 1210 , isolées du même échantillon de yaourt, sont très proches. Elles ont une activité uréasique très forte, des acidités titrables parmi les plus élevées, et des valeurs moyennes pour les autres caractères. Les valeurs de $V m_{1}$ et de $V m_{2}$ des souches CNRZ 1156 et 1204 sont les plus élevées, mais les acidités titrables après $4 \mathrm{~h}$ d'incubation sont parmi les plus faibles.

L'axe $F_{3}$ met en opposition les souches CNRZ 1198, 1157, 1202, 1211 qui ont une forte production d'acétaldéhyde, et les souches CNRZ 1197, 1206 qui en produisent des quantités très faibles. Les autres souches forment un ensemble étendu dans l'espace défini par les 3 axes. L'absence de groupes de souches bien délimités est aussi illustrée par les représentations graphiques sous forme d'étoiles (fig 4), dont la forme est différente pour chaque souche à l'exception des souches CNRZ 1209 et 1210, qui apparaissent identiques. II faut toutefois noter que la souche CNRZ 1209 possède un plasmide que la souche CNRZ 1210 ne possède pas (A Zourari, résultats non publiés). 
Projection du nuage de points sur les axes

factoriels

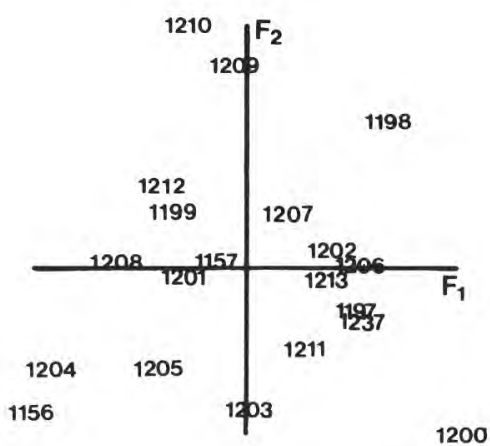

Projection des variables sur le plan factoriel

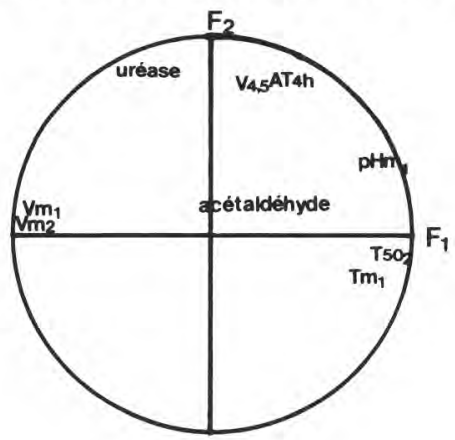

Projection du nuage de points sur les axes

factoriels

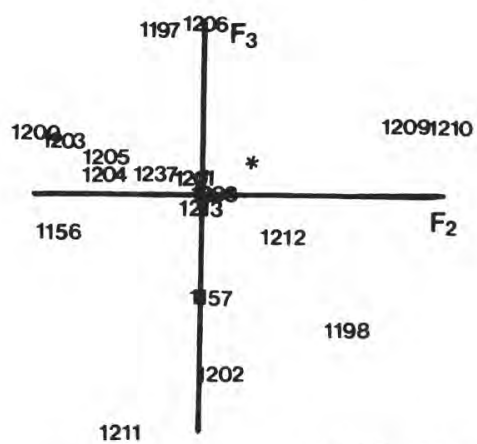

$*: 1199,1207$

Projection des variables sur le plan factoriel

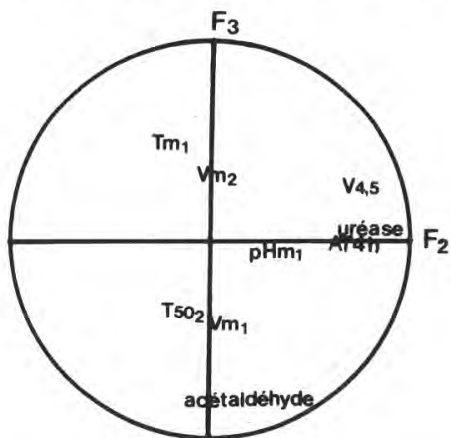

Fig 3. Analyse en composantes principales pour les souches de $S$ salivarius subsp thermophilus. AT: acidité titrable acquise; $V 4,5$ : viscosité à $\mathrm{pH} 4,5$.

Principal component analysis for $\mathrm{S}$ salivarius subsp thermophilus strains. AT: acquired titratable acidity; V4,5: viscosity measured at $\mathrm{pH} 4.5$.

\section{DISCUSSION}

Les valeurs de l'acidité titrable acquise servent le plus souvent à l'évaluation des propriétés d'acidification des bactéries lactiques. L'acidité titrable en fin de croissance et le temps nécessaire pour atteindre une acidité titrable de 40 à $50^{\circ} \mathrm{D}$ (Bouillanne et Desmazeaud, 1980) ou un $\mathrm{pH}$ de 5,5 (Bergère, 1968) sont proposés pour comparer des souches. Ce dernier critère pose le problème du choix de la valeur de l'acidité titrable (ou du $\mathrm{pH}$ ) à atteindre. De plus, de nombreux paramètres interviennent dans l'estimation des propriétés acidifiantes des bactéries lactiques, par exemple la température d'incubation (ACcolas et al, 1977; Martley, 1983; Béal et al, 1989; Chamba et Prost, 1989), le lait utilisé et surtout son traitement thermique (Accolas et Auclair, 1970; Chamba et Prost, 1989), l'état physiologique des cellules de l'inoculum et leur concentration (Chamba 


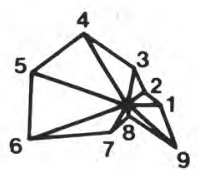

1197

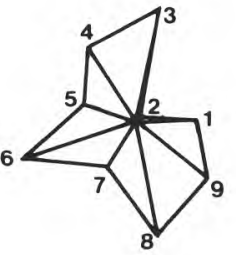

1198

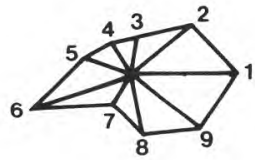

1199

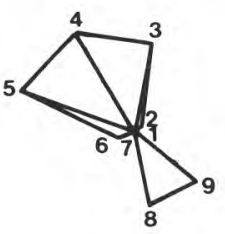

1200

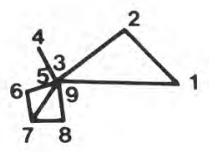

1156

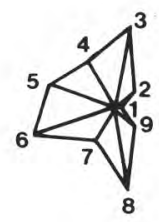

1237

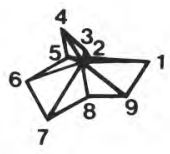

1201

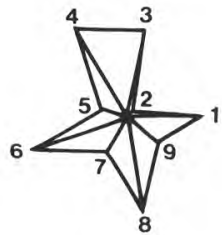

1202

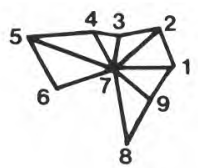

1203

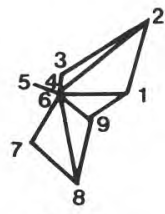

1204

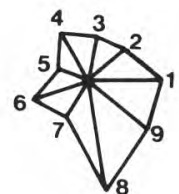

1157

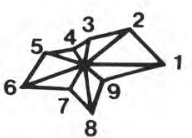

1205

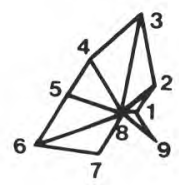

1206

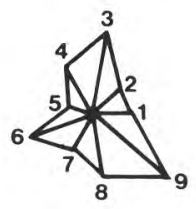

1207

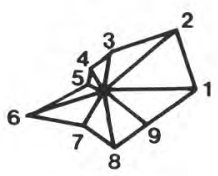

1208

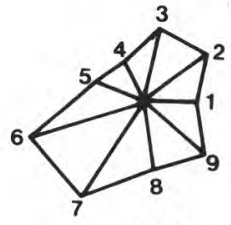

1209

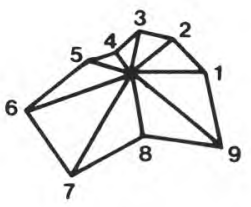

1210

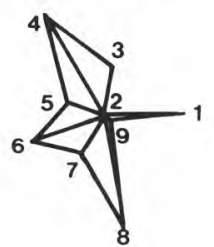

1211

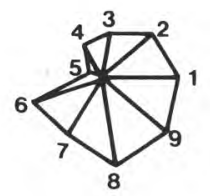

1212

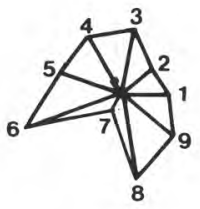

1213

Fig 4. Représentation sous forme d'étoile des souches de $S$ salivarius subsp thermophilus. 1: $V m_{1} ; 2$ : $\mathrm{Vm}_{2} ; 3: \mathrm{pHm}_{1} ; 4: \mathrm{Tm}_{1} ; 5: \mathrm{pH}^{2} \mathrm{O}_{2} ; 6$ : acidité titrable après $4 \mathrm{~h}$ d'incubation; 7: activité uréasique; 8: acétaldéhyde à $\mathrm{pH} 5 ; 9$ : viscosité à $\mathrm{pH} 4,5$.

Star graphics for $\mathrm{S}$ salivarius subsp thermophilus strains. 1: $\mathrm{Vm}_{1} ; 2: \mathrm{Vm}_{2} ; 3: \mathrm{pHm}_{1} ; 4: \mathrm{Tm}_{1} ; 5: \mathrm{pH}_{2}$; 6: titratable acidity after $4 \mathrm{~h}$ incubation; 7: urease activity; 8: acetaldehyde at $\mathrm{pH} 5 ; 9$ : viscosity at $\mathrm{pH}$ 4.5. 
et Prost, 1989). Chaque souche a un comportement particulier vis-à-vis de chacun de ces paramètres, et la comparaison des résultats obtenus par différents auteurs sur les mêmes souches est pour cette raison difficile.

Nous avons suivi l'évolution de l'acidité titrable et du $\mathrm{pH}$ au cours de la croissance dans le lait pour comparer les souches. La mesure du $\mathrm{pH}$ remplace en effet de plus en plus celle de l'acidité titrable, car elle est facile à exécuter et ne s'affecte que d'un faible coefficient de variation (Jomier, 1987).

L'évolution du pH au cours de la croissance de $S$ salivarius subsp thermophilus dans le lait, traduit l'effet global de deux activités opposées : celle de la production d'acide qui tend à abaisser le $\mathrm{pH}$, et celle de l'activité uréasique, qui tend à le remonter. La présence de deux $V m$ résultant de l'activité uréasique de cette bactérie (Spinnler et Corrieu, 1989) complique la comparaison. Elle a été également observée par Famelart et Maubois (1988). Les coefficients de corrélation entre l'activité uréasique et les paramètres de l'acidification sont très faibles (autour de $-0,5$ ). Les conditions de mesure de cette activité enzymatique, très éloignées du milieu lait, pourraient expliquer ces résultats.

II faut noter que l'activité uréasique semble être un caractère général de $S$ salivarius subsp thermophilus (Miller et Kandler, 1967; Tinson et al, 1982; Juillard et al, 1988), ainsi que des souches de $S$ salivarius d'origine orale (Sissons et al, 1989). Ainsi, l'utilisation du caractère "uréase négatif" pour la description de cette espèce n'apparaît pas opportune, malgré la suggestion de Farrow et Collins (1984).

La variation du $\mathrm{pH}$ et l'extrapolation des valeurs de l'acidité titrable sont affectées par le pouvoir tampon du lait, qui est maximal entre $\mathrm{pH} 5$ et 6 . II devient alors difficile d'évaluer l'effet concomitant de la libération d'ammoniaque et de gaz carbonique, qui ont justement lieu dans ces zones de $\mathrm{pH}$. De plus, il faut également tenir compte de la production d'acide lactique et de la solubilisation du $\mathrm{Ca}^{2+}$ colloïdal (Jenness et al, 1974).

Pour la caractérisation des streptocoques, il convient donc de tenir compte à la fois de l'activité uréasique et des vitesses d'acidification, pour éviter de sous-estimer les propriétés acidifiantes d'une souche qui aurait une activité uréasique élevée.

La corrélation étroite entre $\mathrm{Vm}$ et la valeur correspondante de T50 est un point intéressant, déjà observé par Spinnler et Corrieu (1989). Cela pourrait résulter de la sensibilité des bactéries à une quantité donnée d'acide lactique, atteinte plus rapidement quand $V m$ est plus élevée.

Le dosage d'acétaldéhyde, composé principal de l'arôme typique du yaourt, permet d'évaluer le caractère aromatique des souches (Pette et Lolkema, 1950; Dumont et Adda, 1973; Law, 1981; Kurmann, 1983). Nous avons observé, comme certains auteurs (Hamdan et al, 1971; Tealdo et al, 1989), une chute de la quantité d'acétaldéhyde à basse température, apparemment indépendante de la souche (forte corrélation linéaire entre la quantité d'acétaldéhyde mesurée avant et après conservation). Une explication serait le passage d'une quantité d'acétaldéhyde en phase vapeur, en raison de sa grande volatilité et de l'acidité du milieu. II n'y a apparemment pas d'alcool-déshydrogénase (Lees et Jago, 1976; Marshall et Cole, 1983; Raya et al, 1986), ce qui explique que nous n'ayons pas mis en évidence d'augmentation de la quantité d'éthanol pendant la conservation (résultats non montrés).

Une caractérisation globale des souches sur la base de critères d'intérêt technologique fait appel à plusieurs para- 
mètres, qui doivent être considérés simultanément. Une stratégie de sélection peut aussi porter sur des caractères particuliers intéressant un certain type de fabrication. Nous avons cherché une présentation des données facilitant la comparaison rapide des souches par l'ensemble de leurs caractères et leur regroupement par comportement similaire. Ainsi, le remplacement d'une souche par une autre ayant des caractères analogues devient facile, ce qui est par exemple très utile à la suite d'une attaque phagique. II est aussi possible de remplacer une souche par une autre, qui possède une propriété particulière différente. L'ACP utilisée pour la classification des souches tient compte des trois premiers axes, ce qui est indispensable pour la sélection des souches sur la base des trois caractéristiques principales : l'acidification, la viscosité et l'arôme. Notre travail montre que les souches de streptocoques ne peuvent pas être regroupées facilement sur la base de ces trois critères. Autrement dit, il existe une grande diversité de propriétés parmi les souches examinées. Notre objectif étant de constituer un ensemble de souches de référence, dans un premier temps nous n'y avons pas inclus des souches industrielles. II est alors difficile de comparer les souches de ce travail avec les souches utilisées dans l'industrie, sans les tester dans les mêmes conditions, avec les mêmes méthodes, ce qui serait une suite logique et intéressante de la présente étude.

Les représentations graphiques de différents individus sous forme d'étoiles, dont les branches correspondent aux différentes variables étudiées, sont souvent utilisées dans l'évaluation organoleptique des produits (Stone et al, 1974; Robinson, 1988). Les résultats obtenus pour la caractérisation des souches sont plutôt satisfaisants. La forme de l'étoile attribuée à chaque souche permet de comparer rapidement ses caractéristiques globales à celles des autres souches (comparaison de la forme des étoiles) et de savoir quel caractère diffère (longueur relative des branches). Il est même possible de modéliser la comparaison en définissant un profil maximal et un profil minimal (étoiles type) qui donnent la marge à l'intérieur de laquelle doivent se trouver les souches intéressantes pour une fabrication particulière. La superposition des étoiles type et celles des souches examinées permettra alors de savoir rapidement si une souche convient ou non aux besoins d'une fabrication donnée, et quels sont ses défauts.

Les deux méthodes d'exploitation des résultats proposées peuvent aussi être utiles dans le cas d'une collection de souches. Chaque nouvel isolat peut en effet être comparé aux autres et classé dans la collection. En conclusion, nous considérons ces méthodes comme des outils puissants, qui devraient se révéler d'une grande utilité dans le domaine de la sélection de souches, pour l'industrie comme pour la recherche.

\section{REMERCIEMENTS}

Le présent travail a bénéficié d'un financement de la Communauté Économique Européenne (Programme BAP-CEE $n^{\circ}$ 0144F). Nous remercions G Corrieu (Laboratoire du génie des procédés biotechnologiques agro-alimentaires, INRA, Grignon), qui nous a permis d'effectuer au sein de son laboratoire le suivi automatique du $\mathrm{pH}$. Nous remercions également C Bouillanne (Station de recherches laitières, INRA, Jouy-en-Josas), de nous avoir fourni le matériel indispensable pour certaines etudes, ainsi que J Carballo Garcia (Université de Léon, Espagne) pour l'aide qu'il nous a apportée dans la réalisation des mesures de viscosité. Nos remerciements sont aussi adressés à JP Accolas et à J Cerning pour la lecture critique d'une version de ce texte. 


\section{RÉFÉRENCES}

Accolas JP, Auclair J (1970) Détermination de l'activité acidifiante des suspensions concentrées congelées de bactéries lactiques. Lait $50,609-626$

Accolas JP, Bloquel R, Didienne R, Régnier J (1977) Propriétés acidifiantes des bactéries lactiques thermophiles en relation avec la fabrication du yoghourt. Lait 67, 1-23

Béal C, Louvet P, Corrieu G (1989) Influence of controlled $\mathrm{pH}$ and temperature on the growth and acidification of pure cultures of Streptococcus thermophilus 404 and Lactobacillus bulgaricus 398. Appl Microbiol Biotechnol 32, 148-154

Becker RA, Chambers JM, Wilks AR (1988) The New $S$ Language. A programming environment for data analysis and graphics. AT \& T Bell Laboratories, Wadsworth \& Brooks. Cole Advanced Books \& Software, Pacific Grove, California

Bergère JL (1968) Production massive de cellules de streptocoques lactiques. I. Méthodes générales d'étude et facteurs de la croissance de Streptococcus lactis souche C10. Lait 48, 1-11

Bouillanne C, Desmazeaud MJ (1980) Étude de quelques caractères de souches de Streptococcus thermophilus utilisées en fabrication de yoghurt, et proposition d'une méthode de classement. Lait 60, 458-473

Bouillanne C, Desmazeaud MJ (1981) Classement de souches de Lactobacillus bulgaricus selon quelques caractères utilisés en fabrication du yoghurt. Association avec Streptococcus thermophilus. Sci Aliments 1, 7-17

Cerning J, Bouillanne C, Desmazeaud MJ, Landon $M$ (1986) Isolation and characterization of exocellular polysaccharide produced by Lactobacillus bulgaricus. Biotechnol Lett 8 , 625-628

Cerning J, Bouillanne C, Desmazeaud MJ, Landon M (1988) Exocellular polysaccharide production by Streptococcus thermophilus. Biotechnol Lett 10, 255-260

Chamba JF, Prost F (1989) Mesure de l'activité acidifiante des bactéries lactiques thermophiles utilisées pour la fabrication des fromages à pâte cuite. Lait 69, 417-423
Corrieu G, Spinnler HE, Picque D, Jomier Y (1988) Procédé de mise en évidence et de contrôle de l'activité acidifiante d'agents de fermentation dans des bains de fermentation, et dispositif pour sa mise en ceuvre. Brevet FR n ${ }^{\circ} 880456$

Degorce-Dumas R, Goursaud J, Leveau JY (1986) Analyse de composés volatils du yaourt par chromatographie en phase gazeuse-espace de tête (headspace). Ind Agro-Aliment 8, 805-808

Desmazeaud MJ (1989) Influence des traitements technologiques sur les bactéries lactiques. Implications technologiques. In: Les laits fermentés. Actualité de la Recherche. John Libbey Eurotext Ltd, London

Driessen FM (1988) New technology developments in the manufacture of fermented milk. Bull Féd Int Lait 227, 129-137

Dumont JP, Adda J (1973) Méthode rapide d'étude des composés très volatils de l'arôme des produits laitiers. Application au yoghourt. Lait 53, 12-22

Famelart MH, Maubois JL (1988) Comparaison de l'évolution de l'indice de réfraction et de la viscosité au cours de la gélification lactique du lait. Lait 68, 1-12

Farrow JAE, Collins MD (1984) DNA base composition, DNA-DNA homology and long-chain fatty acid studies on Streptococcus thermophilus and Streptococcus salivarius. J Gen Microbiol 130, 357-362

Hamdan IY, Kunsman JE Jr, Deane DD (1971) Acetaldehyde production by combined yogurt cultures. J Dairy Sci 54, 1080-1082

Jenness R, Shipe WF, Sherbon Jr JW (1974) Physical properties of milk. In: Fundamentals of Dairy Chemistry (BH Webb, $\mathrm{AH}$ Johnson \& JA Alford, eds) Avi Publishing Company Inc, Westport

Jomier Y (1987) Estimation de l'activité de ferments lactiques. Étude de différents paramètres, mise au point de méthodes de détermination rapide de l'activité. Mémoire de DEA de Génie Enzymatique, Bioconversion, Microbiologie, Université de Technologie de Compiègne, France

Juillard V, Desmazeaud MJ, Spinnler HE (1988) Mise en évidence d'une activité uréasique chez Streptococcus thermophilus. Can J Microbiol 34, 818-822 
Kurmann JA (1983) Die Joghurtkultur, deren Selektion auf die Produktionseigenschaften im Betriebslaboratorium und deren gärungstechnischer Einsatz in der Joghurt-Fabrikation. Dtsch Milchwirtsch 20, 658-660

Kurmann JA (1984) Aspects of the production of fermented milks. Bull Féd Int Lait 179, 16-28

Law BA (1981) The formation of aroma and flavour compounds in fermented dairy products. Dairy Sci Abstr 43, 143-154

Lees GJ, Jago GR (1976) Acetaldehyde: an intermediate in the formation of ethanol from glucose by lactic acid bacteria. J Dairy Res 43, 63-73

Marshall VM, Cole WM (1983) Threonine aldolase and alcohol dehydrogenase activities in Lactobacillus bulgaricus and Lactobacillus acidophilus and their contribution to flavour production in fermented milks. J Dairy Res 50, 375-379

Martley FG (1983) Temperature sensitivities of thermophilic starter strains. $N Z J$ Dairy Sci Technol 18, 191-196

Miller I, Kandler O (1967) Eiweißabbau und Anreicherung freier Aminosäueren durch Milchsäurebakterien in Milch. III. Die Anreicherung von freien Aminosäuren durch Streptobakterien und Streptokokken. Milchwissenschaft 22, 608-615

Pette JW, Lolkema H (1950) Yoghurt. I. Symbiosis and antibiosis in mixed cultures of $L b$ bulgaricus and Sc thermophilus. Neth Milk Dairy J 4, 197-208
Raya RR, Manca De Nadra MC, Pesce De Ruiz Holgado A, Oliver G (1986) Acetaldehyde metabolism in lactic acid bacteria. Milchwissenschaft 41, 397-399

Robinson RK (1988) Cultures for yogurt - their selection and use. Dairy Ind Int 53, 15-17, 19

Sissons $\mathrm{CH}$, Loong PC, Hancock EM, Cutress TW (1989) Electrophoretic analysis of urea in Streptococcus salivarius and in saliva. Oral Microbiol Immunol 4, 211-218

Spinnler HE, Corrieu G (1989) Automatic method to quantify starter activity based on $\mathrm{pH}$ measurement. J Dairy Res 56, 755-764

Stone H, Sidel J, Oliver S, Woolsey A, Singleton RC (1974) Sensory evaluation by quantitative descriptive analysis. Food Technol 28, 24-34

Tealdo E, Tutta C, Barcarolo R, Castioni R, Faccin M, Fellin A, Gaburro L, Tapparo M (1989) Physico-chemical and organoleptic characterization of yogurt. Latte 14, 153-161

Terzaghi BE, Sandine WE (1975) Improved medium for lactic streptococci and their bacteriophages. App/ Microbiol 29, 807-813

Tinson W, Broome MC, Hillier AJ, Jago GR (1982) Metabolism of Streptococcus thermophilus. 2. Production of $\mathrm{CO}_{2}$ and $\mathrm{NH}_{3}$ from urea. Aust J Dairy Technol 37, 14-16

Zourari A (1991) Caractérisation de bactéries lactiques thermophiles isolées à partir de yaourts artisanaux grecs. Thèse de Doctorat, Paris-Grignon, France 